\title{
Commentary on "Asynchronous Preparation of Tonally Fused Intervals in Polyphonic Music” by David Huron
}

\author{
JAMES WRIGHT[1] \\ Carleton University
}

\begin{abstract}
In his most recent contribution to an important and well-framed series of papers on auditory grouping and the perception of polyphonic music, David Huron seeks further evidence for his working hypothesis that J.S. Bach controls the employment of perfect consonances by desynchronizing their onsets, thereby inhibiting the perceptual fusion of polyphonic voices. However the more novel contribution of the present study would seem to be its demonstration that Bach also regulates the employment of dissonant tones by consistently desynchronizing their onsets. This finding provides the first concrete evidence for Wright and Bregman's otherwise untested hypothesis that the control and salience of dissonance in polyphonic music are closely related to principles of auditory stream segregation (Wright, 1986; Wright \& Bregman, 1987).
\end{abstract}

Submitted 2008 March 10; accepted 2008 April 24.

KEYWORDS: auditory stream segregation, auditory grouping, onset synchrony, consonance, dissonance

IT is well known that studies of visual pattern organization far outstripped related studies in auditory perception for most of the twentieth century. Nonetheless the early Gestalt psychologists left us with a tantalizing set of hypotheses about auditory grouping that were investigated with rigor by later twentiethcentury researchers such as Leon van Noorden (1975) and Albert Bregman (1990). Building upon these foundational studies and the work of pioneering empirical musicologists such as Jay Dowling (1973), Diana Deutsch (1999), Rudolf Rasch (1978, 1981), and Stephen McAdams (1984), David Huron has doggedly sought to shed light on the ways in which principles of auditory pattern organization can inform our understanding of compositional practices and time-tested music-theoretical precepts, across a broad range of musical repertories. In particular, two sets of empirical research questions have been among Huron's most abiding concerns. He has investigated the grouping factors involved in the perception of independent voices in polyphonic music, and the ways in which grouping factors influence our perception of consonance and dissonance. The present study is the most recent in an important and well-framed series of papers in which Huron has brought a powerful battery of methodological tools to bear on both of these topics (Huron, 1989a, 1989b, 1991, 1993, 1995, 2001).

Restricting himself to an examination of Johann Sebastian Bach's fifteen two-part inventions and twenty-six three-part fugues (WTC I and II), Huron sets out to determine whether Bach manipulates onset synchrony in consistent and predictable ways relative to his employment of perfect consonances, imperfect consonances, and dissonances. Huron hypothesizes that if voice independence is an aesthetic ideal for Bach, we might expect him to control the employment of intervals exhibiting the highest degrees of harmonicity (i.e., the perfect consonances) by desynchronizing their onsets, thereby inhibiting the perceptual fusion of polyphonic voices. At the same time, echoing Wright (1986), Wright and Bregman (1987), and Bregman (1990), Huron also hypothesizes that if the control of perceived dissonance is an aesthetic ideal, we might expect Bach to regulate the employment of intervals exhibiting the lowest degrees of harmonicity (i.e., the dissonances) by desynchronizing their onsets, such that the fusion of the dissonant tones is inhibited, and the salience of their dissonance thereby suppressed.

Huron's robust statistical results appear to corroborate both of these seemingly contradictory hypotheses. Employing the Humdrum score-analysis toolkit (Huron, 1995), Huron produces compelling evidence that, in the repertories under examination, Bach shows a strong tendency to employ perfect consonances in asynchronous pattern contexts.[2] Equally conclusive is his finding that Bach shows a 
strong tendency to present dissonances in asynchronous pattern contexts. Finally, Huron shows that Bach tends to employ imperfect consonances in synchronous pattern contexts. Taken together, these results confirm and expand upon findings reported in an earlier study (Huron, 1991), in which Huron characterized Bach's statistical choice of harmonic intervals as an attempt to balance the dual goals of maximizing tonal consonance (by minimizing dissonance), while also maximizing voice independence by controlling the onsets of tones forming perfect consonances, and thereby avoiding tonal fusion.

At this point, I wish to point out a minor irritant in Huron's opening paragraph. While it is clear that "tonal fusion is the tendency for two or more harmonically related tones to fuse and form a single auditory image," onset synchrony is not, as Huron claims, "the tendency for two or more tones having coincident onsets to evoke a single auditory image" (p. 11). Degree of onset synchrony is an acoustic factor that has been shown to influence the perceptual independence of tones embedded in auditory streams (McAdams \& Bregman, 1979; Vos, 1995). As such, onset synchrony has no tendency whatsoever. Similarly, from the time of the classic early studies by Stumpf (1890), 'harmonicity' (defined in roughly Pythagorean terms) has been identified as the primary acoustic factor giving rise to a perceptual phenomenon known as 'tonal fusion.' In short, 'tonal fusion' refers to the formation of a unified percept in the perceptual domain, whereas 'onset synchrony' refers to a pattern variable in the acoustic domain. While Huron's readership will have no difficulty in making this distinction, the failure to differentiate sharply between perceptual and acoustic domains too often gives rise to imprecision and confusion in the discussion of psychoacoustic findings.

It could be argued that Huron overstates his reading of experimental data presented in graphical form by Joos Vos (1995). Huron asserts that Vos's study demonstrates that the amount of asynchrony required to achieve perceptual segregation of two pure tones "is nearly twice as large for just intervals as it is for mistuned intervals" (p. 12). Indeed this assertion would seem to support the hypothesis that the justly-tuned intervals are more highly fused than the unjust intervals, and therefore are more resistant to segregation. Under closer scrutiny, however, Vos's findings appear not to be quite so compelling. In his experimental paradigm, perceptual segregation was associated with the extent to which listeners could determine whether, in sequences of two successive two-tone dyads, the pitch of the upper tone was the same or different. The results that Vos reports-for the just intervals there were $81 \%$ correct responses in the asynchronous condition and $68 \%$ correct responses in the synchronous condition, while for the mistuned intervals there were $80 \%$ correct responses in the asynchronous condition and $73 \%$ correct responses in the synchronous condition - constitute a difference that Huron describes as "nearly twice as large" (13\% versus $7 \%$, respectively). Given both the scale and absolute magnitude of these differences, 'gee whiz' graphs of the kind Huron presents here can be misleading (Schneider \& Shanteau, 2003). The statistical significance of Vos's experimental findings appear to be somewhat weaker than Huron suggests.

Throughout this study, Huron's primary goal is to seek evidence for his working hypothesis that Bach controls the employment of perfect consonances by desynchronizing their onsets, thereby inhibiting the perceptual fusion of polyphonic voices. While the present paper corroborates and expands upon earlier findings that support this hypothesis (e.g., Huron 1991), the more novel contribution of the present study would seem to be its demonstration that Bach also regulates the employment of dissonant tones by consistently desynchronizing their onsets. This finding provides the first concrete evidence for Wright and Bregman's otherwise untested hypothesis that the control and salience of dissonance in polyphonic music are closely related to principles of auditory stream segregation (Wright, 1986; Wright \& Bregman, 1987).

Wright and Bregman suggest that a gradual loosening of onset/offset constraints on the pattern context of dissonant simultaneities runs parallel to the oft-cited historical 'emancipation of dissonance' that characterizes the evolution of harmonic language in Western music between the sixteenth and twentieth centuries.[3] Further support for this hypothesis might be sought by analyzing historical trends in the control of onset synchrony with respect to harmonic dissonance. Using methodologies similar to those employed by Huron in the present study, it is to be hoped that later polyphonic repertories (the fugues of Shostakovich, for example) might be subjected to similar investigation.

Huron concludes his paper by pointing out that his results "contribute to the view that compositional practice in polyphonic music shows significant adaptations consistent with the extant research pertaining to auditory stream segregation” (p. 19). Detractors of empirical musicology sometimes argue that such findings are self-evident. But as Huron and others continue to provide empirical evidence in support of music theoretical propositions-and, almost as often, counterevidence refuting some of the traditional claims of music theory-they allow us to speak with new and hard-won understanding, 
precision, authority, and intelligence about the pattern variables involved in the perception of complex musical textures.

\section{NOTES}

[1] School for Studies in Art and Culture: Music Carleton University, Ottawa, Canada.

[2] Huron (p. 19) points out that the perfect unison appears to constitute a special case. The evidence reveals that, relative to the perfect fifth, perfect fourth, and perfect octave, the asynchronous onset of the perfect unison is less likely to increase stream segregation, perhaps especially in keyboard works.

[3] See, for example, Schoenberg (1975, p. 217). It should also be noted that Wright and Bregman also assert that, by the twentieth century, the polyphonic sonance spectrum encompasses not just the consonances and dissonances, but also 'non-sonances,' simultaneities embedded in pattern contexts where the tones are so highly segregated as to give rise to no salient harmonic percept whatsoever.

\section{REFERENCES}

Bregman, A.S. (1990). Auditory scene analysis: The perceptual organization of sound. Cambridge, MA: MIT Press.

Deutsch, D. (ed.) (1999). The psychology of music (2nd ed.). San Diego: Academic Press.

Dowling, W.J. (1973). The perception of interleaved melodies. Cognitive Psychology, 5, 322-337.

Huron, D. (1989a). Voice denumerability in polyphonic music of homogeneous timbres. Music Perception, 6(4), 361-382.

Huron, D. (1989b). Voice segregation in selected polyphonic keyboard works by Johann Sebastian Bach. Unpublished doctoral dissertation, University of Nottingham.

Huron, D. (1991). Tonal consonance versus tonal fusion in polyphonic sonorities. Music Perception, 9(2), 135-154.

Huron, D. (1993). Note-onset asynchrony in J.S. Bach's two-part inventions. Music Perception, 10(4), 435444.

Huron, D. (1995). The Humdrum toolkit reference manual. Menlo Park, CA: Center for Computer Assisted Research in the Humanities.

Huron, D. (2001). Tone and voice: A derivation of the rules of voice leading from perceptual principles. Music Perception, 19(1), 1-64.

McAdams, S. (1984). Spectral fusion, spectral parsing, and the formation of auditory images. Unpublished doctoral dissertation, Stanford University, Stanford, CA.

McAdams, S., \& Bregman, A.S. (1979). Hearing musical streams. Computer Music Journal, 3(4), 26-43, 60,63. 
Rasch, R.A. (1978). The perception of simultaneous notes such as in polyphonic music. Acustica, 40, 2133.

Rasch, R.A. (1981). Aspects of the perception and performance of polyphonic music. Doctoral dissertation. Utrecht: Elinkwijk BV.

Schneider, S., \& Shanteau, J. (Eds.) (2003). Emerging perspectives in judgment and decision making. Cambridge: Cambridge University Press.

Schoenberg, Arnold (1975). Style and idea. Leonard Stein (ed.), Leo Black (trans.). Berkeley/Los Angeles: University of California Press.

Stumpf, C. (1890). Tonpsychologie (2 vols.) Leipzig: Verlag S. Hirzel.

van Noorden, Leon P. A. S. (1975). Temporal coherence in the perception of tone sequences. Doctoral dissertation. Eindhoven: Technical University Eindhoven.

Vos, J. (1995). Perceptual separation of simultaneous complex tones: The effect of slightly asynchronous onsets. Acta Acustica, 3(5), 405-416.

Wright, J.K. (1986). Auditory object perception: Counterpoint in a new context. Unpublished masters thesis, McGi11 University, Montreal, Quebec, Canada.

Wright, J.K. \& Bregman, A.S. (1987). Auditory stream segregation and the control of dissonance in polyphonic music. Contemporary Music Review, 2, 63-93. 